\title{
Spannende Hypothese
}

_ Die „Neuro“ hat mich schon immer fasziniert. Obwohl es vor 13 Jahren im Praktikum nicht ganz optimal lief: Eine Patientin mit Hemiparese rutschte mir zwischen Bett und Rollstuhl weg, und ein Patient mit Femoralis-Parese kam auf der Krankenhaustreppe zwischen zweitem und erstem Stock ins Wanken. Da diese beiden therapeutischen Fehlschläge nicht die letzten waren, zog ich einen Schlussstrich unter die Neuro. Zurück bleiben die Erinnerungen an Rigor, Freezing und spastische Muster und viele offene Fragen. Meine „Neue“ hieß übrigens „Ortho“. Mit ihr lief es deutlich besser.

- Seit ich bei physiopraxis arbeite, sind die Neuro und ich uns wieder näher gekommen. Vor ein paar Wochen unterhielt ich mich mit der Bobath- und NOI-Instruktorin Nora Kern und wurde hellhörig. Ihre Theorie ist, dass die spastischen Muster das lädierte Nervensystem vor zu viel Spannung schützen. Für mich als Freund der Ortho ist das eine spannende Hypothese, greifbar und nachzuvollziehen. Ob sie sich belegen lässt, muss sich zeigen. Darüber nachzudenken lohnt sich auf jeden Fall, finde ich. Aber bilden Sie sich Ihre Meinung selbst: Das Interview mit Nora Kern lesen Sie ab Seite 30.

_ Dieses Thema haben wir auch intensiv im Redaktionsteam diskutiert, in dem wir übrigens gerade wieder Verstärkung bekommen haben: Die Physiotherapeutinnen Kathrin Bauer und Astrid Nedbal sind unsere Neuen - und eine echte Bereicherung.

Eine spannende Lektüre wünscht Ihnen

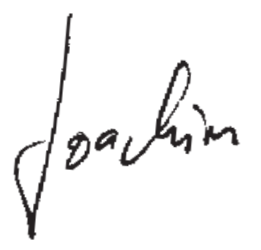<smiles>CC(C)C1CCCC1</smiles>

Das physiopraxis-Team (v. I.): Rosi Haarer-Becker, Tanja Wilhelm, Annette Alkemade, Elke Baumann, Joachim Schwarz, Maria Czyganowski, Markus Böggemann, Astrid Nedbal, Fritz Koller, Kathrin Bauer

\section{PHYSIOBONUS}

physiobonus heißen die Gewinnspiele in physiopraxis. Wer teilnehmen möchte, nutzt das Formular unter www.thieme.de/ physioonline in der Rubrik physioexklusiv. Und das gibt es in dieser Ausgabe zu gewinnen:

Kursplatz

1 Kursplatz

„PT auf der Slackline“ Seite 22

1 Kursplatz „Kraniosakrale Osteopathie“

Seite 56

Bücher

2-mal

„Frühphase Schlaganfall“ Seite 22

3-mal „Mobilisation des Nervensystems“

Seite 32

3-mal „Kindesmissbrauch“ Seite 36

3-mal „Gesundheitsförderung am Arbeitsplatz“

Seite 42

und außerdem

1 Boxsack

mit Handschuhen

Seite 11

1 Slackline

mit Baumschutz

Seite 26

2 Antistressbälle 